\title{
Temps, température et variations saisonnières
}

\section{Marcel Fournier}

\section{OpenEdition}

\section{Journals}

Édition électronique

URL : http://journals.openedition.org/ress/387

DOI : $10.4000 /$ ress.387

ISSN : 1663-4446

\section{Éditeur}

Librairie Droz

\section{Édition imprimée}

Date de publication : 1 mars 2004

Pagination : 117-127

ISBN : 2-600-00941-8

ISSN : 0048-8046

Référence électronique

Marcel Fournier, "Temps, température et variations saisonnières », Revue européenne des sciences sociales [En ligne], XLII-129 | 2004, mis en ligne le 05 novembre 2009, consulté le 19 avril 2019. URL http://journals.openedition.org/ress/387 ; DOI : 10.4000/ress.387 


\section{Marcel FOURNIER}

\section{TEMPS, TEMPÉRATURE ET VARIATIONS SAISONNIÈRES}

Temps, températures et variations saisonnières : ce sont des éléments centraux de la vie de nos sociétés contemporaines, même si nous nous ne sommes plus aussi directement «soumis » à la «nature» que nos prédécesseurs. Dans un pays dit «tempéré» comme le Canada, toutes les conservations quotidiennes portent sur «le temps qu'il fait». Le journal La Presse consacre même un éditorial à la question. «Déjà, nous étions obsédés par le temps qu'il fait: les récriminations contre les conditions météo constituent probablement le second sport national des Québécois après le hockey. Avez-vous remarqué le nombre de minutes à la télé, le nombre de pages dans le journal qui sont consacrés au temps qu'il fait?» ${ }^{\text {. }}$

Temps, température et variations saisonnières: qu'il s'agisse de modes de vie, de régimes politiques ou de criminalité, les observateurs de la vie sociale n'ont pu ignorer l'effet de la localisation géographique et par là de la température. La démocratie, comme le puritanisme,n'est-elle pas un produit des régions plus nordiques de l'Europe? On parle de l' «effet Montesquieu $»^{2}$. Montesquieu établit en effet une relation causale entre, d'un côté. différents éléments d'ordre géographique ou morphologique, tels que le climat, la géographie, le volume et la distribution de la population, le genre de vie, et de l'autre, les formes de gouvernement: le despotisme est à la démocratie ce qu'un espace étendu avec climat excessif est à un pays de faible étendu avec climat froid au nord et un sol pauvre. On pourrait aussi parler de l' «effet Le Play »: le développement de l' «initiative personnelle» a aussi ses causes géographiques et climatiques. Les criminologues, en particulier ceux de l'École italienne, ont analysé les liens entre criminalité et météorologie.

«Beau temps pour le crime !», écrit encore aujourd'hui un jeune criminologue dans sa thèse de doctorat: la violence, qu'il s'agisse de voies de fait ou d'agressions sexuelles, augmente avec la hausse de la température. Le phénomène s'expliquerait par la théorie des activités routinières: les gens sortent davantage de chez eux les beaux jours, ce qui entraîne une augmentation des interactions entre les gens et par le fait même un accroissement des risques de conflits interpersonnels. Il ne semble cependant pas que le suicide soit sensible aux variations saison-

Katia Gagnon, «Vive la canadienne», La Presse, Jeudi 2 octobre 2003, A14.

Pierre Bourdieu, «Le Nord et le Midi. Contribution à l'analyse de l'effet Montesquieu», Actes de la recherche en sciences sociales, 35, novembre 1980, p. 21-25. 
nières: il n'y a pas plus de suicide par mauvais temps et aucune saison en particulier n'entraînerait un plus grand nombre de suicides ${ }^{3}$.

Temps, température et variations saisonnières: ce sont là trois thèmes qui ont intéressé, voire fasciné Durkheim et deux de ses proches collaborateurs, Henri Hubert et Marcel Mauss. Le notion de temps (à la fois linéaire et cyclique) était au centre de leur programme de recherche en sociologie de la connaissance. La question du climat a soulevé l'agacement de Durkheim dans son travail sur le suicide. Les variations saisonnières ont été l'objet de l'essai fameux que publie Marcel Mauss en collaboration avec Henri Beuchat dans L'Année sociologique.

Temps, température et variations saisonnières: tels sont les objets d'un ouvrage que Philippe Besnard consacre en 1987 aux Moeurs et humeurs au fil des saisons. L'anthropologue Westermarck soutenait déjà en 1892 dans son History of Human Marriage,l'idée d'une saison sexuelle qu'il situait au printemps. Le livre qui s'adresse à un grand public tente d'apporter une réponse aux questions que nous nous posons tous: quels sont les moments de l'année propices aux amours, aux rencontres, aux mariages, aux naissances? Quels sont les moments à risque pour les maladies, les déprimes, les accidents, les vols, les crimes, les suicides, les violences privées ou publiques?

Notre objectif est de remettre en perspective cet ouvrage de Philippe Besnard en revenant aux travaux classiques de l'Ecole sociologique française, principalement à ceux de Durkheim et de Mauss. Le sous-titre de notre article pourrait être: «L'École sociologique française: d'Émile Durkheim à Philippe Besnard».

\section{À CHAQUE SAISON, SON CONTINGENT DE SUICIDE, DIXIT DURKHEIM}

«Chaque saison apporte son contingent de suicides», écrit Durkheim dans Le Suicide $e^{4}$. Contagion, épidémie, contingent: Durkheim parle comme un pastorien, n'hésitant pas, lorsqu'il aborde l'influence des facteurs cosmiques, à utiliser le mot « germe »: il en est, selon lui, de l'aptitude pour le suicide comme d'un germe, il n' «éclôt» que dans des conditions physiques ou matérielles particulières. Parmi les facteurs cosmiques qui retiennent l'attention des spécialistes, par exemple Morselli en Italie, il y a le climat et la température saisonnière. Durkheim infirme d'abord l'hypothèse selon laquelle il y aurait une relation entre le climat tempéré et la tendance au suicide. Les facteurs seraient plutôt sociaux: en Italie, pour ne prendre que cet exemple, les suicides suivent le mouvement scientifique, artistique et économique qui, Rome devenant la capitale, se déplace du Nord au Centre. Il n'y a pas non plus, comme l'a établi Montesquieu, de relation entre les pays brumeux et froids et la tendance au suicide. L'influence de la température saisonnière est l'inverse de ce qu'on pense habituellement: ce n'est pendant ni l'automne ni l'hiver (deux périodes de l'année, qui avec le ciel sombre et l'aspect

Mathieu Robert-Sauvé, «Beau temps pour le crime!», Forum, vol 38, no 3, 8 septembre 2003, p. 1. L'auteur de l'étude, Étienne Blais, a dépouillé plus de 160000 rapports de policie de la Communauté urbaine de Montréal entre 1995 et 1998. Les résultats doivent paraître dans la Revue internationale de criminologiste comparée et de police scientifique.

$4 \quad$ Émile Durkheim, Le suicide, Paris, PUF, p. 87. 
désolé de la nature, disposerait à la rêverie, à la tristesse, bref à la mélancolie), mais pendant la «belle saison» que le suicide atteint son maximum: la chaleur serait un «stimulant du suicide $»^{5}$. Les faits que fournissent les criminologues italiens Ferri et Morselli et qui portent sur différents pays européens apparaissent « incontestables». Par ailleurs, les «diverses observations » que rapporte Brierre de Boismont attribuent aux températures extrêmes une influence directe: par exemple il y a eu dans l'armée française une augmentation des suicides pendant l'expédition de Napoléon en Égypte et aussi pendant la retraite de Moscou.

«La question reste donc entière», conclut Durkheim qui ajoute immédiatement: «C'est à l'analyse des données statistiques qu'il faut en demander la solution $»^{\circ}$. Chaque analyse statistique (ou construction de tableau) constitue, pour reprendre son expression, une expérience, qui permet de confirmer ou d'infirmer telle ou hypothèse ou «interprétation des faits». Pour la France, l'Italie et la Prusse, Durkheim compare le nombre de suicides selon les saisons (printemps et automne) et selon les mois, avec température moyenne pour chaque saison ou chaque mois dans chacun des pays), et, une fois mise en lumière les faiblesses et les contradictions des analyses de ses collègues italiens, il poursuit sa propre analyse en construisant une nouvelle variable, la longueur moyenne des journées en France; il établit qu'il y a «une relation entre la marche du jour et celle du suicide », l'augmentation des suicident allant de pair avec l'allongement des jours. Le suicide varie donc tout au long de l'année comme la longueur du jour. On attribue souvent à Durkheim la paternité de cette thèse, mais celui-ci n'est pas le premier à avoir mis en rapport le suicide et la longueur du jour ${ }^{7}$.

Durkheim compare aussi le nombre de suicides selon le moment ou l'heure de la journée pour arriver au constat suivant: «Le jour est plus fécond en suicides que la nuit $»^{8}$. Une telle variation dans la journée ne s'explique pas, selon lui, par des changements de température mais des changements d'activités: il y a des périodes où les affaires sont plus actives, les relations humaines plus fréquentes et la vie sociale plus active". Par exemple, sur l'heure du midi, il y a une «accalmie», «le suicide s'arrête un instant». Il y a enfin des variations selon le jour de la semaine. Gabriel Tarde n'a pas dit autre chose: dans son ouvrage La criminalité comparée (1886), il sépare l'intensification de la vie sociale de l'action de la température.

Tout semble donc s'expliquer par «l'effervescence de la vie sociale» et «l'intensité de la vie publique», comme lorsqu'on compare la distribution mensuelle des suicides en compagne et en ville. Durkheim construit même un indicateur d'intensité de la vie sociale, qui est le volume des communications et des rapports intersociaux mesuré par les recettes des grandes lignes de train pour la grande vitesse, selon les saisons, pour l'année $1887^{10}$. La dimension technologique appa-

\footnotetext{
Ibid., p. 86.

Ibid., p. 91.

Massimo Borlandi, «Lire ce que Durkheim a lu. Enquête sur les sources statistiques et médicales du Suicide», in Massimo Borlandi et Mohamed Cherkaoui (dir.), Le suicide, un siècle après Durkheim, Paris, PUF, 2000, p. 37.

8 Émile Durkheim, Le suicide, op. cit., p. 99.

9 Ibid., p. 100.

10 Les données sont tirées du Bulletin du ministère des Travaux publics.
} 
raît ici importante. Durkheim tient aussi compte, pour les villes européennes, de l'éclairage artificiel qui écourte la période d'obscurité. Une conclusion s'impose: la «marche du suicide» est affectée directement non pas par des facteurs physiques ou cosmiques, mais par des conditions sociales. Toute la question est de savoir «comment la vie collective peut avoir cette action ${ }^{11}$.

\section{LES VARIATIONS SAISONNIÈRES ${ }^{12}$}

Émile Durkheim signe avec son neveu Marcel Mauss un essai sur les «Formes primitives de classification » qui paraît dans L'Année sociologique en 1904. Cette étude, fort ambitieuse, ouvre une voie nouvelle à la sociologie de la connaissance: il est en effet possible, comme ils le souhaitent, d'analyser d'autres «fonctions ou notions fondamentales de l'entendement humain» (les idées d'espace, de temps, de cause, de substance, les différentes formes de raisonnement, etc.). Tel est, comme le soulignera Mauss, le programme de L'Année sociologique: «Nous isolâmes tout de suite, d'accord avec Durkheim, le problème de la raison ». Ce sera l'étude des représentations religieuses abordées de «bien des côtés»: nombre, cause (mémoire sur les origines des pouvoirs magiques); espace, temps (mémoire sur l'origine de la notion de temps); âme; monde (notion d'orientation), genre, espace. Et Mauss conclura: «Cet effort est l'un des plus philosophiques qui aient été tentés par une école. Durkheim l'a poussé à fond d'un point de vue dogmatique dans ses Formes élémentaires de la vie religieuse» ${ }^{13}$.

L'intention générale est de mettre en lumière une relation d'interdépendance et d'interpénétration entre des ordres foncièrement différents de faits sociaux, par exemple l'organisation sociale et les représentations collectives. Mauss poursuit ses travaux dans cette direction avec son essai sur «Les variations saisonnières ». En 1903-1904, Mauss consacre ses conférences à l'École pratique à l'étude de documents ethnographiques concernant les rapports de la famille et de la religion en Amérique du Nord; il invite Henri Beuchat à présente quatre leçons sur des questions d'ethnographie pure qui ont trait aux Esquimaux, à leurs migrations, à leur morphologie sociale, à leur technologie ${ }^{14}$. Beuchat est alors secrétaire-trésorier de la Revue des études américaines et prépare avec M. Le Souëf un ouvrage illustré sur l'art mexicain. Mauss remarque l'habileté technique de son élève et lui confie la tâche de confectionner, pour L'Année sociologique, les tables et les index. En 1904-1905, la conférence porte toujours sur les Esquimaux: étude critique des documents récemment publiés et analyse de la question du totémisme. Beuchat explique des textes danois et prépare, en collaboration avec son professeur, un travail sur «les rapports entre la morphologie sociale, saisonnière, des Eskimos et leurs phénomènes religieux et juridiques $\gg^{15}$.

\footnotetext{
Ibid., p. 106.

Voir aussi notre étude Marcel Fournier, Marcel Mauss, Paris, Fayard, 1994.

13 Marcel Mauss, «L'œuvre de Mauss par lui-même» (1930), Revue française de sociologie, 20 (1), 1979, p. 218.

${ }_{14}$ Annuaire de l'École pratique des hautes études, section des sciences religieuses, 1904, p. 59.

15 Ibid., 1905, p. 43.
} 
Mauss et Beuchat publient en 1906 dans L'Année sociologique un mémoire sous le titre «Essai sur les variations saisonnières des sociétés Eskimos. Étude de morphologie sociale». Selon le plan original, la participation de Mauss devait être secondaire, mais rapidement la relation s'inverse et le professeur doit «reprendre entièrement le travail $»^{16}$. La collaboration de Beuchat se limite à la préparation d'une partie du mémoire et, en raison de ses qualités de dessinateur, à la réalisation graphique de plans de maisons et de cartes.

Pour Mauss, il s'agit de poursuivre, sur un autre terrain et à un autre niveau, I'étude qu'il a effectuée avec Durkheim sur «Les classifications » et dans laquelle il a démontré que «la mentalité des tribus inférieures reflète directement leur constitution anatomique». D'ordre méthodologique, l'enjeu est d'établir une correspondance entre, d'un côté, «la vie sociale, sous toutes ses formes, morale, religieuse, juridique, etc.», et, de l'autre, son substrat matériel, c'est-à-dire «la masse, la densité, la forme et la composition des groupes humains ${ }^{17}$, Mauss reprend ici une hypothèse que Durkheim a lui-même cherché à vérifier, d'abord dans De la division du travail social et ensuite dans Le Suicide: dans un cas, l'évolution du droit pénal et du droit civil est fonction du type morphologique des sociétés; dans l'autre, les croyances individualistes se développent ou s'affaiblissent suivant le degré d'intégration des groupes sociaux.

La démarche qu'emprunte Mauss n'est pas comparative; son étude des sociétés esquimaudes se présente comme une «expérience cruciale», sur un seul cas. Son intention est non pas de «rassembler, en une monographie descriptive, les particularités diverses des peuples Eskimos», mais d'en dégager l'unité: pour ces populations de chasseurs et de pêcheurs qui ne connaissent ni organisation tribale stricte ni territoire délimité, cette unité réside dans 1 '«établissement» (settlement), dans l'habitat commun de groupes de familles Sous ce rapport, les Esquimaux offrent un champ d'étude d'autant plus privilégié que leur morphologie n'est pas la même aux différents moments de l'année: en été, il y a dispersion de l'habitat et dispersion des familles s'abritant sous la tente; en hiver, il y a «resserrement» autour de la longue maison où logent plusieurs familles. Après les voyages et les migrations lointaines vient le temps de l'immobilité relative de l'hiver.

Ces variations saisonnières traduisent manifestement une adaptation aux contraintes environnementales: obligation de se disperser pour faire la chasse aux caribous et aux bœufs musclés, I'été; nécessité d'unir les forces aux points de capture des morses, I'hiver. Mais ce déterminisme écologique fournit une explication incomplète, puisqu'il ne permet pas de rendre compte de l'intensification de la vie collective pendant les longs mois d'hiver. Les deux saisons s'opposent tout autant sur le plan de la religion et de la famille que sur celui du régime des biens et de l'organisation politique L'une est symbolisée par les «peaux de rennes», et l'autre par les «peaux de morses». L'été se caractérise par les pratiques minimales du culte domestique, la famille nucléaire et l'appropriation

\footnotetext{
Marcel Mauss, «L'œuvre de Mauss par lui-même» (1930), op. cit., p. 211.

7 Marcel Mauss (en collaboration avec Henri Beuchat), «Essai sur les variations saisonnières des sociétés Eskimos », L'Année sociologique, 9, 1906, in Marcel Mauss, Sociologie et anthropologie, Paris, PUF, 1966, p. 475.
} 
familiale ou individuelle des biens; l'hiver, par l'activité religieuse intense, les nombreuses fêtes, la parenté collective et le partage communautaire de la nourriture et des biens. D'un côté, I'individualisme (et l'égoïsme) et, de l'autre, le collectivisme: "A une communauté réelle d'idées et d'intérêts dans l'agglomération dense de l'hiver, à une forte unité mentale religieuse et morale, s'opposent un isolement, une poussière sociale, une extrême pauvreté morale et religieuse dans l'éparpillement de l'été.» ${ }^{18} \mathrm{~A}$ la fois économique et sexuel, avec échange des femmes, le «communisme» exerce ici une fascination d'autant plus grande qu'il «montre à quel degré d'unité morale parvient [en hiver] la communauté eskimo »: c'est «le moment d'apogée» de la vie sociale.

La «curieuse alternance» qu'observe Mauss n'est pas spécifique aux Esquimaux, on la retrouve aussi chez les Indiens de la côte du Pacifique, par exemple chez les Kwakiutl, qu'a étudiés Franz Boas, et chez des populations pastorales des montagnes de l'Europe. Mauss pousse son analyse comparative jusqu'à «nos sociétés occidentales » où il observe les «mêmes oscillations »: en ville, période d'alanguissement durant l'été et jusqu'à la fin de l'automne, puis l'hiver, intensification des activités et des relations;à la campagne, situation inverse: en hiver, dispersion et torpeur, et en été, fêtes, grands travaux et grandes débauches ${ }^{19}$.

Il s'agit donc d'une loi générale: la vie sociale «passe par des phases successives et régulières d'intensité croissante et décroissante, de repos et d'activité, de dépense et de réparation». Parce qu'elle exerce une «violence» sur les consciences, la vie en commun n'est possible qu'à la condition que les membres d'un groupe puissent «s'y soustraire en partie».

Outre le grand rythme des saisons, il existe des oscillations à l'intérieur de chaque saison, de chaque mois, de chaque semaine, de chaque jour. Cette question du rythme de la vie collective intéresse aussi Henri Hubert, qui, dans son étude sur l'idée du temps dans la religion et la magie, cherche à expliquer la formation du calendrier.

L'«Essai sur les variations saisonnières» peut paraître schématique. Au moment de sa parution, le débat est ouvert. E Sidney Hartland croit que la vie des collectivités n'est pas aussi simple que celle des oiseaux; il reproche à Mauss d'avoir établi «entre l'été et l'hiver une différence d'organisation sociale plus grande que ne le permettent les faits». Pour cet anthropologue anglais, fort sympathique à L'Année sociologique, il n'est cependant pas impossible que «le rythme de dispersion et de concentration, de vie individuelle et de vie collective corresponde à une loi plus générale». Toute la question est de savoir si «les variations sont entièrement provoquées par des changements morphologiques $»^{20}$. L'anthropologue anglais R. R. Marett, ardent défenseur de la psychologie sociale, reproche aussi à Mauss d'être allé trop loin dans les «one-sided explanations of morphological derivation $»^{21}$. Mauss tiendra à défendre son point de vue:

\footnotetext{
Ibid., p. 470.

Ibid., p. 473.

20 E. Sidney Hartland, compte rendu de L'Année sociologique, 9, 1906, in Folklore, vol. XXVII, March 1907, p. 100.

${ }^{21}$ R. R. Marett, «A Sociological View of Comparative Religion», The Sociological Review, vol. 1, 1908, p. 52.
} 
«Je n'attribue nullement aux facteurs morphologiques une importance prépondérante. Mais je croyais avoir tant développé, dans mes travaux antérieurs, le point de vue physiologique ou [ill.], que je ne pensais pas qu'il fût nécessaire de m'expliquer là-dessus. Je n'ai d'ailleurs pas besoin de vous dire que j e ne crois en l'existence, dans la société, que d'hommes matériellement disposés et affectés dans le temps et dans l'espace, d'une part, de phénomènes de conscience communs et sociaux, d'autre part. [...] Je définis, comme vous, tous les phénomènes qui ne concernent pas la morphologie ou les réactions de la psychologie sur la morphologie, en termes de conscience, de psychologie».

$\mathrm{Au}$ moment où il rédige l' «Essai sur les variations saisonnières », Mauss élargit, dans ses cours, son champ d'intérêt américaniste et analyse des textes ethnographiques portant sur les sociétés américaines du Nord-Ouest (les Kwakiutl, les Bella Coola, etc.), en particulier ceux de Boas, dont il critique les interprétations. Pendant L'année scolaire 1905-1906, il soumet à l'étude comparative le potlatch, une institution qui «affecte et domine tous les phénomènes sociaux de ces tribus ». L'un de ses auditeurs, Marius Barbeau, un jeune diplômé en droit et boursier canadien, prépare à Oxford, sous la direction de R. R. Marett, une thèse intitulée The Totemic System of North Western Indian Tribes of North America (1910). Il sera l'un des premiers membres de l'équipe d'ethnologie dirigée par Edward Sapir, qui, en 1913-1914, mettra sur pied la première expédition scientifique canadienne dans l'Arctique. A cette expédition, dirigée par N. U. Stefanson, participeront deux «anthropologistes», Diamond Jenness (de Nouvelle-Zélande) et Henri Beuchat. Pour Beuchat, qui est chargé de porter son attention à la langue, aux manières, aux coutumes et aux croyances religieuses des populations de l'Arctique ${ }^{22}$, ce sera la chance de devenir anthropologue professionnel, d'observer pendant quatre ans les Esquimaux et de «connaître les peuples avec lesquels il vit en imagination depuis longtemps». Dans une longue lettre à Mauss, dans laquelle il décrit les préparatifs, il conclura: «Tout augure bien pour le succès de l'expédition.» ${ }^{23}$ Mais dès le début de l'expédition, en janvier 1914, à la suite du naufrage du chalutier Karkluck, il meurt à l'île de Wrangell, de faim et de froid ${ }^{24}$.

Un autre étudiant de Marcel Mauss à l'École Pratique des Hautes Éudes va consacrer sa vie à l'étude des Esquimaux. Il s'agit de Paul-Émile Victor qui effectue de nombreux et longs séjours au Groenland. Cette expérience le rend, écrit-il, «farouchement partisan du travail seul,isolé, assimilé dans la population qu'il désire étudier $»^{25}$. En annexe de son journal Banquise, il présente un «tableau ethnographique des Eskimo d'Angmagssalik», dont les deux aspects principaux sont 1) la position dans l'espace, et 2) la position dans le temps. Pour ce qui est de la position dans le temps, Victor distingue les variations saisonnières et les variations annuelles, et il reprend, tout en apportant des nuances, l'analyse de Mauss

\footnotetext{
22 Diamond Jenness, The Life of the Cooper Eskimos. A Report of the Canadian Arctic Expedition, 1913-1918, Ottawa, 1922, p. 9.

23 Lettre d'Henri Beuchat à Marcel Mauss, Victoria, 16 juin 1913.

24 Marcel Mauss, «In Memoriam. L'œuvre inédite de Durkheim et de ses collaborateurs » (1925), in Marcel Mauss, Euvres, t. 3, Paris, Éditions de Minuit, 1969, p. 489.

25 Paul-Henri Victor, «Raison d'un hivernage solitaire», in Banquise, p. 350.
} 
sur les Variations saisonnières ». Il y a bien, note Victor, deux morphologies bien distinctes: la morphologie d'été et la morphologie d'hiver. L'été se caractérise par le nomadisme, l'utilisation du oumiak (grand bateau pour la famille ramé par les femmes) et du kayak comme moyens de transport, et la tente comme habitat. L'hiver, c'est le sédentarisme: peu de déplacements familiaux, transport en trầneau, et comme habitat, les maisons de pierres et de terre.

Dans son analyse fort schématique, Paul-Émile Victor ajoute plusieurs précisions: le troc se fait l'été (jusqu'à récemment), et les grandes fêtes ont lieu l'été, plus particulièrement lors de la pêche aux caplans. Et dans un tableau synoptique intitulé «Technomorphologie des eskijmo d'Angmagssalik», il montre que le phoque est un élément d'unité: on en tire la lumière, la chaleur,la cuisson, la nourriture, les vêtements, l'habitat, et les moyens de transport. L'été et l'hiver vont s'opposer non seulement comme s'opposent le nomadisme (habitat transportable) et le sédentarisme (habitat fixe), mais aussi comme s'opposent l'eau (transport essentiellement maritime) et la terre (transport terrestre).

\section{MEURS ET HUMEURS DES FRANÇAIS AU FIL DES SAISONS}

«Il y a une saison pour toute chose, un temps pour enfanter et un temps pour mourir [...]» (L'Ecclésiaste)? Un temps pour mourir? Philippe Besnard cite cet extrait dans son ouvrage, Mours et humeurs des Français au fil des saisons, paru chez Balland en 1989. Que fait ce durkheimologue averti dans le domaine dees mœurs et des humeurs? Besnard a un intérêt scientifique pour l'étude de la mode, qui l'a mené vers les prénoms, et chaque année, il fait paraître chez le même éditeur en collaboration Un prénom pour toujours, qui deviendra un bestseller sous le titre de La cote des prénoms. Les saisons, les prénoms: voilà de bien curieuses préoccupations. Ce sont là, peut-on penser, des préoccupations peu sérieuses. Philippe Besnard y met pourtant tout le sérieux et toute la rigueur qu'on lui connaît dans ses travaux d'histoire de la sociologie,et il réunit une documentation considérable et souvent inédite. Il s'inscrit ainsi en filiation avec l'École sociologique française.

Pour Mours et humeurs des Français au fil des saisons, Besnard ajoute en annexe une soixantaine de graphiques qui présentent diverses courbes saisonnières (selon les mois de l'année) et sur lesquels s'appuie une grande partie de l'ouvrage. Tout y est ou presque: la température, la longueur du jour l'ensoleillement, les cycles de la vie (naissance, conceptions, mariages, mortalité), la vie sexuelle, l'activité économique (chômage, grèves, ventes des journaux ou des automobiles, loto, les crimes (viols, attentats à la pudeur, cambriolages, vols d'automobile). Sans oublier évidemment les suicides. À ces données statistiques s'ajoutent de nombreuses informations sur le folklore et les traditions populaires: les fêtes païennes et chrétiennes, les huit temps des rites (solstices, etc.) rites et finalement les rythmes d'aujourd'hui (semestres, sept fois sept semaines, vacances).

Contrairement à l'idée que l'on se fait de la société moderne et qui tend à niveler les mois et les jours, Besnard montre que les Français restent farouchement «attachés à l'alternance de temps forts et de temps morts, aux rites saison- 
niers qui cadencent et qui brisent l'écoulement monotone des jours ordinaires $»^{26}$. Il y a bel et bien des saisons privilégiées pour la sexualité: l'une à la frontière du printemps et de l'été, en juin, et l'autre à la frontière de l'été et de l'automne, en septembre. Tout cela relève, selon Besnard, de l'occasion et de la programmation, de la disponibilité et du calcul.

Le mariage est aussi un phénomène saisonnier, plus fortement aujourd'hui qu'hier. Tout se passe pendant la belle saison, principalement à la fin du mois de juin. Deux mois ont une mauvaise réputation: c'est le mois de novembre, qui est placé sous le signe de la mort, et le mois de mai, qui est placé sous le signe de la virginité (La Vierge Marie). La Noce de mai est, selon un dicton populaire, une «noce de mort». De telles variations, encore fortes aujourd'hui, ne s'expliquent ni par des facteurs culturels ou religieux ni par des calculs utilitaires, Tout se passe, note Besnard, comme si «nos mœurs matrimoniales avaient besoin de cette respiration périodique. Voilà qui suggère que les rythmes saisonniers de notre vie, individuelle et collective, répondent à une nécessité profonde $»^{27}$.

Et si le cœur a ses raisons, le corps a ses saisons. Il y a un mouvement saisonnier de la mort avec un pic des décès fin janvier-début février et un creux fin aoûtdébut septembre.» La morte saison est, conclut Besnard, la saison des morts ${ }^{28}$. Tout cela donne l'impression d'un rythme naturel qui suit, avec un peu de retard, celui de la température. Certaines causes de décès sont plus visiblement saisonnières, par exemple la grippe en hiver. Besnard observe par ailleurs que la période charnière d'août-septembre est, sous ce rapport privilégiée. Et se rappelant que cette période se caractérise aussi par une grande pulsion sexuelle, il conclut: «On meurt moins quand on donne la vie et inversement». Besnard s'amuse à contester les résultats douteux des études d'astrologie médicale. Mais il note la relation étrange observée entre naissance en hiver et prédisposition pour la schizophrénie, et il s'intéresse aussi aux travaux qui relève de la chronobiologie humaine qui est à l'écoute des rythmes biologiques internes (sur 24 heures ou un an). Aurionsnous des horloges biologiques? Et où seraient-elles logées? Probablement audessus du croisement des nerfs optiques...

Il en serait de même des humeurs qui seraient saisonnières: mélancolie l'automne, langueur l'été, enthousiasme le printemps. On serait, selon les sondages de la SOFRES, plus pessimiste de septembre à décembre, et plutôt optimiste en juin. Mais rien de vraiment concluant. Besnard s'attarde à la séduisante hypothèse du « désordre affectif saisonnier» élaborée par des psychiatres américains : les symptômes de dépression surgissent à l'automne pour disparaître vers avril. Est-ce dû à l'exposition insuffisante à la lumière? Rien n'est encore confirmé.

En bon spécialiste de Durkheim, Besnard ne peut ignorer la question des suicides et de leurs variations selon les saisons. Il n'y a pas de relation entre saison morte ou triste et les suicides. Besnard compare les courbe annuelle du suicide avec celle de la mortalité générale et avec elle des naissances. Paradoxalement, c'est «quand la vie surgit avec le plus de force que l'on y renonce le plus volontiers». Mais il corrige immédiatement: «Les moments où l'on donne la vie sont

\footnotetext{
26 Philippe Besnard, Mæurs et humeurs des Français au fil des saison, Paris, Balland, 1989, p. 8.

27 Ibid., p. 65.

28 Ibid., p. 75.
} 
indépendants de ceux où l'on se donne la mort ${ }^{29}$. Ce qui l'intéresse, c'est la régularité saisonnière des suicides dans le temps: le taux de suicide est plus élevé en juin. On a d'abord pensé à un effet de la chaleur estivale. Durkheim,le premier dès la fin du X1Xe siècle a pourfendu la théorie de la température pour mettre en évidence un facteur proprement social: l'intensité de la vie sociale, qui est plus grande en été. C'est là, note un Philippe Besnard critique, une «interprétation à la fois étrange et rapide», puisqu'elle amène Durkheim à supposer que l'activité agricole commence à s'apaiser dès le mois d'août ${ }^{30}$. Or c'est à l'automne, vers octobre, que l'intensité de la vie diminue.

Durkheim aurait donc été trop expéditif en rejetant tout influence des «facteurs naturels». Or, comme le souligne Besnard, le phénomène du mouvement saisonnier des suicides est universel; «L'acte en apparence leplus personnel, qui est le fruit d'une impulsion individuelle ou d'une délibération intime, est aussi celui dont le rythme saisonnier est le plus universel $\gg^{31}$. Les chiffres sont explicites: marée montante de morts volontaires au printemps, et baisses des eaux autour de décembre. Effet de la luminosité? La pulsion suicidaire est-elle, se demande Besnard, une maladie à «long jour», provoquée par l'accroissement de la lumière? Il y a aussi les rythmes sociaux: les débuts de semaine, avec le recommencement des activités, sont plus propices au suicide. On observe par ailleurs dans plusieurs pays une certaine recrudescence, surtout chez les jeunes et les femmes, des suicides en septembre et octobre. Est-ce l'angoisse des recommencements? Et pour les femmes, y aurait-il, se demande Besnard, une influence du cycle menstruel? Aucune question n'est donc négligée. Besnard n'écarte pas d'entrée de jeu les thèses chronobiologiques même si celles-ci le laissent quelque peu perplexe. «Nos humeurs seraient-elles encore plus insondables qu'inconstantes?», se demande-t-il. Et il conclut: «Cette inconstance n'est peut-être pas sans vertu. Si l'on pouvait saisir les raisons des saisons de la mélancolie faudraitil aussi vouloir en régulariser le cours annuel? On peut préférer les grandes marées des humeurs aux mortes eaux de la tristesse ordinaire $»^{32}$.

Qu'en est-il finalement des violences? Sont-elles saisonnières? Philippe Besnard réunit une quantité impressionnante de données sur les diverses formes de violence: violence routière, violence physique et sexuelle, vols, violences collectives (guerres, coups d'état, émeutes, insurrections). Le sociologue sait se faire tout à la fois démographe, criminologue et politologue. Il montre bien que tout explication d'ordre climatique risque d'être simpliste. Le printemps, période de la montée de sève printanière, est souvent spontanément associé au bouillonnement des passions et aux mobilisations collectives. Mai et juin sont, note Besnard, particulièrement riches en journées insurrectionnelles: la Commune débute en mars. Celui-ci avance prudemment une explication en établissant un rapprochement entre le mouvement saisonnier des révoltes et le calendrier des fêtes populaires traditionnelles: les violences collectives qui appartiennent au répertoire des fêtes sont portées au paroxysme dans les fêtes printanières (qui sont

\footnotetext{
Ibid., p. 88-89.

Ibid.

Ibid., p. 90.

32 Ibid., p. 95-96.
} 
entièrement aminées par les jeunes gens célibataires), lors du Carnaval et des rites de mai. «Ainsi a pu se forger et s'enraciner une tradition séculière de contestation printanière qui a survécu au dépérissement des fêtes saisonnières d'antan $»^{33}$. Aussi ne faut-il pas se surprendre que plus près de nous, la colère estudiantine ait explosée en mai 68, et qu'elle ait été organisée par un «mouvement du 22 mars ». C'est la saison des débordements juvéniles... C'est probablement ce que Philippe Besnard pense de Mai 68.

Temps, température, variations saisonnières. Même dans nos sociétés industrielles avancées, dites sociétés de l'information et de la communication, il y a un temps pour chaque chose. Le temps est une chose si précieuse que certaines grandes villes européennes - Milan, Paris - ont créé des Bureaux du temps dont la responsabilité est coordonner les temps des divers secteurs d'activités (entreprises, garderies, transports, etc). Par ailleurs, les variations de température ne laissent personne indifférent, à un point tel que selon les saisons, on change certaines de ses activités et parfois son mode de vie. L'été tragique 2003 a fait mentir les statistiques sur les mortalités (qui généralement diminuent en juin, juillet et août), mais les douze mille morts en France nous rappellent que la mortalité, surtout chez les personnes âgées, est fonction des écarts de température. Il aurait peut-être suffi d'installer des climatiseurs dans les hôpitaux et dans les foyers pour personnes âgées. La technologie ne réussit cependant que partiellement à réduire l'impact les différences saisonnières, tout comme elle ne nous protège pas des catastrophes (dont les catastrophes climatiques). Parfois la technologie provoque de telles catastrophes... Une chose est certaine: on ne cessera pas de si tôt de parler du temps qu'il fait. On s'inquiète déjà ici et là du réchauffement de la planète, comme si une telle modification des températures risquait de changer profondément les modes et les conditions de vie de millions de gens.

Département de sociologie

Université de Montréal

marcel.fournier@umontreal.ca

${ }_{33}$ Ibid., p. 113. 